\title{
AN EQUIVALENT FORM OF LEVY'S AXIOM SCHEMA
}

\section{MURRAY JORGENSEN}

\begin{abstract}
A generalized notion of ordinal inaccessibility is defined. A characterization of this notion in terms of normal ordinal functions yields as a consequence that a schema analogous to a form of Tarski's Axiom of Inaccessible Cardinals is equivalent to Lévy's axiom schema under the axiom of choice.
\end{abstract}

This note supplies proof to the result announced in [1]. Using elementary ordinal theory alone we derive a schema that is equivalent to that of Lévy in the presence of AC (the axiom of choice) and that has a simple relationship to Tarski's Axiom of Inaccessible Cardinals. R. Montague has obtained another equivalent form of Lévy's schema as a consequence of a model-theoretic lemma proved in Montague [3]. It turns out that our form and Montague's form of the Lévy schema correspond to the two commonly stated forms of Tarski's Axiom of Inaccessible Cardinals. We make use of the notation and definitions of Lévy [2], in particular we borrow the illegitimate convenience of speaking of functions with domain the class of all ordinals. Actually, all our "functions" will be of this kind and instead of ordinal-valued function defined for all ordinals we will write simply function. A canonical example is the ordinal successor function. By weakly (strongly) inaccessible we shall mean im weiteren (im engeren) Sinne unerreichbar in the sense of [4], but inaccessible (unqualified) has the sense of [2].

1. Three schemata. Consider the following three statements:

(1) Every normal function has at least one inaccessible number in its range.

(2) Every normal function has at least one regular number in its range.

(3) Given a function $f$ and an ordinal $\beta$ there is a regular ordinal $\alpha$ greater than $\beta$ such that $\gamma<\alpha$ implies $f(\gamma)<\alpha$.

In Bernays-Godel set theory we can express these statements as single sentences by quantification over "functions," but in ZermeloFraenkel set theory we must regard (1), (2) and (3) as schemata.

(1) is the original axiom schema $M$ introduced in [2] and (2) is a weakening of it. The property

Received by the editors February 16, 1970.

AMS 1969 subject classifications. Primary 0268; Secondary 0430.

Key words and phrases. Axiom schema, fixed point of normal function, inaccessible cardinals, transfinite number, ordinal. 


$$
\gamma<\alpha \text { implies } f(\gamma)<\alpha
$$

enjoyed by $\alpha$ in (3) may be regarded as asserting the inaccessibility of $\alpha$ with respect to the function $f$. We term this property $f$-inaccessibil$i t y$, so (3), then, asserts the existence of arbitrarily large regular $f$ inaccessible numbers for every ordinal function $f$.

We shall prove the equivalence of (2) and (3) without the aid of the axiom of choice, and the equivalence of all three schemata with that axiom.

\section{A characterization of $f$-inaccessibility.}

LEMMA. Let $\nu$ be any ordinal. If $f$ is a function such that $f(\mu)>\mu$ for every ordinal $\mu$, then there exists a unique normal function $\phi_{f}$ such that

$$
\phi_{f}(\xi+1)=f\left(\phi_{f}(\xi)\right), \quad \phi_{f}(0)=\nu .
$$

Proof. Veblen [5, Theorem 2].

Subject to some natural restrictions on $f$, the regular $f$-inaccessible numbers greater that $\nu$ will be just the regular fixed points of $\phi_{f}$. This follows from the following, more general

THEOREM. If $f$ and $\phi_{f}$ are as in the lemma, and for all ordinals $\eta^{\prime}, \eta$

$$
\eta^{\prime}<\eta \quad \text { implies } f\left(\eta^{\prime}\right) \leqq f(\eta)
$$

then $\zeta$ is $f$-inaccessible and greater than $\nu$ iff there exists a limit ordinal $\lambda$ such that $\zeta=\phi_{f}(\lambda)$.

Proof. As $\phi_{f}$ is normal $\phi_{f}(\zeta) \geqq \zeta$ by $\left[5\right.$, p. 283]. If $\phi_{f}(\zeta)=\zeta$ the result follows as $\eta<\zeta$ implies $\eta+1 \leqq f(\eta)<\zeta$ by the $f$-inaccessibility of $\zeta$, so that $\zeta$ is a limit ordinal. If $\phi_{f}(\zeta)>\zeta$, let $\mu$ be the least ordinal such that $\phi_{f}(\mu)>\zeta$, then $\mu=\mu^{\prime}+1$ for if $\mu$ is a limit ordinal then we can find $\mu^{\prime \prime}<\mu$ such that $\phi_{f}\left(\mu^{\prime \prime}\right)>\zeta$.

Further $\phi_{f}\left(\mu^{\prime}\right)=\zeta$ as if $\phi_{f}\left(\mu^{\prime}\right)<\zeta$ then $\phi_{f}(\mu)=f\left(\phi_{f}\left(\mu^{\prime}\right)\right)<\zeta$ (contradiction).

Finally $\mu^{\prime}$ is a limit ordinal, as if $\mu^{\prime}=\lambda^{\prime}+1$ then $\phi_{f}\left(\lambda^{\prime}\right)<\zeta$ and $\phi_{f}\left(\mu^{\prime}\right)=f\left(\phi_{f}\left(\lambda^{\prime}\right)\right)<\zeta$ (contradiction).

Conversely if $\zeta=\phi_{f}(\lambda)$ where $\lambda$ is a limit ordinal then if $\eta<\zeta$ we can find $\mu<\lambda$ such that $\phi_{f}(\mu)>\eta$, so

$$
f(\eta) \leqq f\left(\phi_{f}(\mu)\right)=\phi_{f}(\mu+1)<\phi_{f}(\lambda)=\zeta
$$

and so $\zeta$ is $f$-inaccessible.

COROLLARY. With the above notation and assumptions on $f, \zeta$ is regular, $f$-inaccessible and greater than $\nu$ iff $\zeta$ is a regular fixed point of $\phi_{f}$. 
If $f$ lacks the properties

$$
\eta<f(\eta), \quad \eta^{\prime}<\eta \Rightarrow f\left(\eta^{\prime}\right) \leqq f(\eta),
$$

we can consider the function $f^{\prime}$ defined by

$$
f^{\prime}(\mu)=\max \left(\sup _{\xi<\mu} f(\xi), \mu+1\right) .
$$

Then $f^{\prime}$ has the required properties and all $f^{\prime}$-inaccessible numbers are $f$-inaccessible.

The equivalence of (2) with (3) is now immediate: if we are granted (2) and given an ordinal $\nu$ and a function $f$, a simple argument like that of $\left[2\right.$, Theorem 1] shows that $\phi_{f^{\prime}}$ has regular fixed points. These are $f$-inaccessible and greater than $\nu$.

If we are granted (3) we must notice that any normal function $\phi$ can be written as $\phi_{f}$ for some $f$, e.g. $f(\xi)=$ the least member of range $\phi$ greater than $\xi$. The above corollary, with (3), yields a regular fixed point of $\phi$.

3. Some consequences. By taking $f$ to be (a) the cardinal successor function, (b) the function $f(\xi)=2^{\xi}$ (cardinal power), we obtain as consequences of our schema (3): (a) the existence of arbitrarily large weakly inaccessible numbers and (b) the existence of arbitrarily large strongly inaccessible numbers (if granted $\mathrm{AC}$ ).

Lévy proves $[2$, p. 228] that (1) is equivalent to the conjunction of (2) with the statement "there exist arbitrarily large inaccessible numbers". As the axiom of choice implies that "strongly inaccessible" and "inaccessible" are the same thing $[2$, p. 226] it is a consequence of the above remark that (1), (2), and (3) are equivalent under this axiom.

4. Closure principles and Montague's schema. Tarski's Axiom of Inaccessible Cardinals can be stated in two forms:

(Closure Form.) For every set $M$ there is a set $R$ such that

(i) $M \in R$,

(ii) $X \in Y$ and $Y \in R$ implies $X \in R$,

(iii) $X \in R$ implies $2^{x} \in R$,

(iv) if $X \subseteq R$ and no function maps $X$ on to $R$, then $X \in R$.

(Inaccessibility Form.) For every cardinal $\beta$ there is a strongly inaccessible cardinal $\alpha$ greater than $\beta$.

In [4], the two forms are shown to be equivalent under the axiom of choice. The closure form actually implies this axiom. Our schema (3) can well be regarded as the "inaccessibility form" of Lévy's 
schema. R. Montague, in [3], considers a schema which can be regarded as a closure form of Lévy's schema:

(4) For every set $A$ there is a set $B$ such that

(i) $A \subseteq B$,

(ii) if $X \subseteq B$ and no function maps $X$ on to $B$ then $\zeta(X) \in B$.

Here $\zeta$ is any term of $Z F$ in which the variable $B$ is not free. It is a consequence of the results of Montague that (4) is equivalent to (1), (2) and (3) under the axiom of choice. Actually Montague proves that a variant of (4) is equivalent to a variant of (1) without AC. It is further pointed out in [3] that (4) yields the closure form of Tarski's axiom upon suitable choice of $A$ and $\zeta$. Hence (4) itself implies AC, and the implication (4) $\rightarrow(3)$ can be proved without invocation of AC. However the reverse implication $(3) \rightarrow(4)$ cannot be established without the axiom of choice for A. Lévy (in a private communication) has supplied me with a simple forcing argument demonstrating the consistency of (2), and hence (3), with the negation of the axiom of choice.

\section{REFERENCES}

1. M. Jorgensen, An equivalent form of Lêvy's axiom schema, Notices Amer. Math. Soc. 16 (1969), 1086-1087. Abstract \#69T-E82.

2. A. Lévy, Axiom schemata of strong infinity in axiomatic set theory, Pacific J. Math. 10 (1960), 223-238. MR 23 \#A1522.

3. R. Montague, Two contributions to the foundation of set theory, Proc. Internat. Congress Logic, Methodology and Philosophy of Science (1960) Stanford Univ. Press, Stanford, Calif., 1962, pp. 94-110. MR 27 \#4748.

4. A. Tarski, Über unerreichbare Kardinalzahlen, Fund. Math. 30 (1938), 68-89.

5. O. Veblen, Continuous increasing functions of finite and transfinite ordinals, Trans. Amer. Math. Soc. 9 (1908), 280-292.

University of British Columbia, Vancouver 8, B. C., Canada 